\title{
FLORA LEÑOSA DEL BOSQUE DE GARÚA DE LA CORDILLERA CHONGÓN COLONCHE, SANTA ELENA - ECUADOR
}

\section{THE WOODY FLORA OF THE DRIZZLE (GARUA) FOREST OF THE CHONGON COLONCHE CORDILLERA, SANTA ELENA - ECUADOR}

\author{
Evelyng Astudillo-Sánchez ${ }^{1}$, James Pérez ${ }^{2}$, Luis Troccoli ${ }^{3}$, Héctor Aponte ${ }^{4}$ y Oscar Tinoco ${ }^{5}$
}

\begin{abstract}
Resumen
La cordillera Chongón Colonche conocida por su alta diversidad y endemismo presenta vacíos de información biológica así como la deforestación que amenazan la biodiversidad del sitio. Se determinó la composición, estructura y diversidad de la flora leñosa en dos localidades de la cordillera, Loma Alta y Dos Mangas, mediante parcelas de 0.1 ha ubicadas a $500 \mathrm{msnm}$. Se identificaron 48 especies, 46 géneros y 28 familias, donde las más representativas fueron Arecaceae y Urticaceae. La estructura estuvo conformada por seis clases diamétricas y siete altimétricas. El endemismo y estado de conservación se congrega en 19 especies (40\%), de las cuales Inga carinata "Guaba", Gustavia serrata "Membrillo", Sorocea sarcocarpa "Tillo" y Randia carlosiana "Cañafito" son las más importantes. La diversidad alfa oscila en rangos medios, donde Loma Alta presentó mayor diversidad promedio (2.40 nats/ind) y menor dominancia promedio (0.13). La tasa de recambio (1.29) fue mayor en Loma Alta y el análisis de ordenación nMDS-ANOSIM-SIMPER estableció diferencias significativas $(\mathrm{R}=0.80, \mathrm{p}<0.01)$ definiendo dos grupos disimiles $(54 \%)$ florísticamente. La diversidad vegetal de los bosques de garúa está compuesta por familias características de los bosques húmedos occidentales y que difieren de la vegetación característica de los Bosques Estacionalmente Secos del Pacífico Ecuatorial, pero ambas regiones florísticas convergen en esta cordillera; por lo que, la conservación del bosque y un manejo efectivo de sus áreas protegidas es imperativo. Los resultados contribuyen al conocimiento local y regional de los bosques húmedos y secos de la costa ecuatoriana.

Palabras clave: Dos Mangas, Loma Alta, composición florística, diversidad alfa y diversidad beta.
\end{abstract}

\begin{abstract}
The Chongon Colonche Mountain Range is located within the Tumbes-Choco-Magdalena region, known for its high diversity and endemism, which faces deforestation and presents biological information gaps as conservation problems. The composition, structure and diversity of the woody flora were determined in two localities, Loma Alta and Dos Mangas, through plots of 0.1 ha located at 500 masl. Forty-eight species, forty-six genera and twenty-eight families were identified, where the most representative were Arecaceae and Urticaceae. The structure was made up of six diametric and seven altimetric classes. Endemism and conservation status congregate in 19 spp. (40\%), of which Inga carinata "Guaba", Gustavia serrata "Membrillo", Sorocea sarcocarpa "Tillo" and Randia carlosiana "Cañafito" are the most important in both categories. The alpha diversity of the garua forests oscillated in medium ranges, where Loma Alta presented higher average diversity (2.40 nats/ind) and lower average dominance (0.13). The turnover rate (1.29) was higher in Loma Alta and the nMDS-ANOSIM-SIMPER ordering analysis established significant differences $(R=0.80$, $\mathrm{p}<0.01)$ defining two dissimilar floristic groups $(54 \%)$. The vegetal diversity of the garua forests is composed of characteristic families of the western humid forests and they differ from the characteristic vegetation of the Seasonally Dry Forests of the Equatorial Pacific region; however, both floristic regions converge in this cordillera. Therefore, forest conservation and effective management in these protected areas is imperative. These results contribute to the local and regional knowledge of the humid and dry forests of the Ecuadorian coast.
\end{abstract}

Key words: Dos Mangas, Loma Alta, floristic composition, alpha diversity, beta diversity.

\section{Introducción}

Los puntos calientes de biodiversidad son regiones biogeográficas que concentran altos niveles de endemismo en plantas y tienen una alta pérdida de hábitat (Mittermeier et al., 2011). A nivel mundial se han identificado 35 puntos calientes de biodiversidad, esto es el $17.3 \%$ de la superficie de la Tierra que alberga el $77 \%$ de todas las plantas endémicas, el $43 \%$ de los vertebrados y el $80 \%$ de todos los anfibios amenazados (Marchese, 2015). El punto caliente de biodiversidad 
Tumbes-Chocó-Magdalena (TCM), conocido como la Ecorregión Terrestre del Chocó-Darién-Ecuador Occidental, se extiende desde Panamá, atraviesa el occidente de Colombia y Ecuador, y termina al Noreste de Perú, conteniendo una variedad de ecosistemas (CEPF, 2005).

La región del Chocó, compartida con Colombia, alberga a bosques húmedos y muy húmedos premontanos reportando 9000 especies de plantas vasculares (2 250 endémicas), 830 de aves (10\% endémicas), 235 mamíferos (25\% endémicas), 350 anfibios $(60 \%$ endémicas) y 210 reptiles $(30 \%$ endémicas) (CEPF, 2005; Palacios \& Jaramillo, 2016). El Chocó Ecuatoriano acoge 6300 especies vegetales (25\% de la flora del país), donde el $20 \%$ es endémica y 142 especies de mamíferos (10\% endémico) (CEPF, 2005). Los Bosques Estacionalmente Secos (BES) en el Neotrópico, acorde a Banda et al. (2016), están definidos por 12 grupos florísticos que se diferencian hasta en un 73\% y el grupo andino exhibe 6 subgrupos heterogéneos florísticamente, donde 2 de estos, Apurimac-Mantaro y Tarapoto-Quillabamba, no tienen protección formal. La región Tumbesina, compartida con Perú, se caracteriza por los BES que corresponden al núcleo florístico conocido como Pacífico Ecuatorial $\mathrm{y}$ son bosques reconocidos por su alta diversidad y endemismos (Linares-Palomino et al., 2010). La región tumbesina alberga 6300 especies de plantas, de las cuales 1290 (20.5\%) son endémicas; 800 especies de aves, de estas $55(7 \%)$ son endémicas; 142 especies de mamíferos, de los cuales 54 (38\%) son endémicas (Paladines, 2003).

En el Ecuador, la región litoral u occidente de Ecuador, se caracteriza por dos grupos disimiles de bosques que difieren en composición florística, diversidad, fitogeografía y condiciones climáticas (Cornejo, 2018); estas características biogeográficas concuerdan con las regiones del Chocó y Tumbes. Los bosques occidentales cubrieron aproximadamente 80 $000 \mathrm{~km}^{2}$ y menos del $6 \%$ corresponden a tres diferentes tipos de vegetación, bosque húmedo tropical (tropical wet forest $-0.8 \%$ ), bosque seco tropical (tropical dry forest - 1\%) y bosque tropical de garúa (tropical moist forest $-4 \%$ ) (Dodson \& Gentry, 1991).

La Cordillera Chongón Colonche (CCHC) se ubica en el centro oeste de la región litoral, abarca un área de $95 \mathrm{Km}$ en sentido este-oeste (Bonifaz \& Cornejo, 2004) recorriendo las provincias geográficas de Guayas, Santa Elena y Manabí, y en ella confluyen dos regiones biogeográficas, Chocó y Tumbes (Valverde et al., 1991). En la CCHC se presenta un bioclima de semiárido a subhúmedo y se describen bosques húmedos en las partes altas, a partir de los $400 \mathrm{msnm}$, y bosques secos en las partes bajas (MAE, 2013). La CCHC enfrenta dos problemas de conservación, la deforestación y la carencia de información, que dificultan la gestión ambiental de la biodiversidad presente.
La deforestación fue confirmada por Dodson \& Gentry (1991) al referirse que el $90 \%$ del bosque de las tierras bajas y estribaciones del Pacífico ecuatoriano debajo de los $900 \mathrm{msnm}$ ha sido transformado a zonas de cultivos y plantaciones siendo la principal amenaza en la costa. La región litoral presenta una tasa de deforestación de $1.9 \%$ y en ella seis de sus catorce ecosistemas están en peligro o vulnerables porque han perdido entre el $50-70 \%$ de su cobertura original (Sierra, 2018), donde Santa Elena y Guayas fueron las provincias más afectadas para el período 2000-2008 (MAE, 2012).

Por otra parte, a pesar de las diferentes contribuciones en ecología vegetal por diversos autores (Paladines 2003; Pennington et al., 2004; Aguirre \& Delgado, 2005; Leal-Pinedo \& Linares-Palomino, 2005; Vázquez et al., 2005; Aguirre et al., 2006a b; Marcelo-Peña et al., 2007; García-Villacorta, 2009; Pennington et al., 2009; Linares-Palomino et al., 2010; Espinoza et al., 2012; Jadán et al., 2014; Muñoz et al., 2014; Portillo-Quintero et al., 2015; Escribano-Avila, 2016; Eguiguren-Velepucha et al., 2016; EduardoPalomino et al., 2017; Escribano-Avila et al., 2017) quienes han enfocado sus investigaciones en los BES del Pacífico Ecuatorial, persiste la carencia de información en la costa ecuatoriana y en particular en la CCHC así lo enuncia Cuesta et al. $(2013 ; 2017)$ al mencionar que prevalecen vacíos biológicos ubicados en la transición entre sistemas húmedos del Pacífico con los estacionales y deciduos de la costa central.

Por tal motivo, el objetivo de este estudio es analizar la composición, estructura y diversidad de la vegetación leñosa presente en dos localidades de la cordillera Chongón Colonche a $500 \mathrm{msnm}$, a fin de estrechar el vacío de información biológica para la costa ecuatoriana y fortalecer la gestión de los recursos forestales y la sostenibilidad del área.

\section{Materiales y métodos}

Área de Estudio

El área de estudio está restringida hacia dos localidades de la provincia de Santa Elena que poseen un área protegida. La comuna Loma Alta $\left(01^{\circ} 52^{\prime} \mathrm{S}, 80^{\circ}\right.$ $\left.38^{\prime} \mathrm{O}\right)$ posee el Bosque Protector Loma Alta y el transecto se ubicó a $466 \mathrm{msnm}$; entretanto, la comuna Dos Mangas $\left(02^{\circ} 07^{\prime} \mathrm{S}, 80^{\circ} 18^{\prime} \mathrm{O}\right)$ forma parte del Bosque Protector Chongón Colonche (BPCHC) y el transecto se localizó a 589 msnm (Figura 1).

Las condiciones climáticas varían acorde a la orografía, es decir, dependen del relieve y altitud en la CCHC. En las partes altas de la cordillera, la temperatura promedio es de $21^{\circ} \mathrm{C}$, con una máxima de $36^{\circ} \mathrm{C}$, durante el día y la precipitación alrededor de 800 - $1200 \mathrm{~mm}$ en la estación lluviosa, mientras que la temperatura desciende hasta los $18^{\circ} \mathrm{C}$ en las noches y la precipitación llega hasta $1080 \mathrm{~mm}$ en la estación de garúa (Bonifaz \& Cornejo, 2004; MAE, 2017). Por el contrario, en las laderas y zonas pobladas de la 

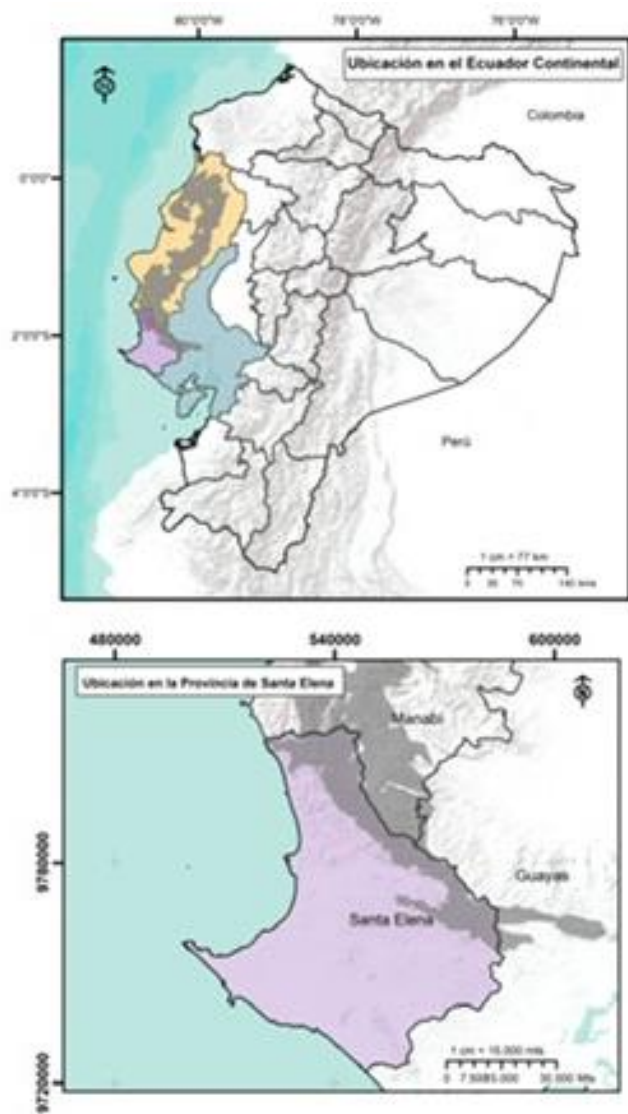

CORDILLERA CHONGÓN COLONCHE
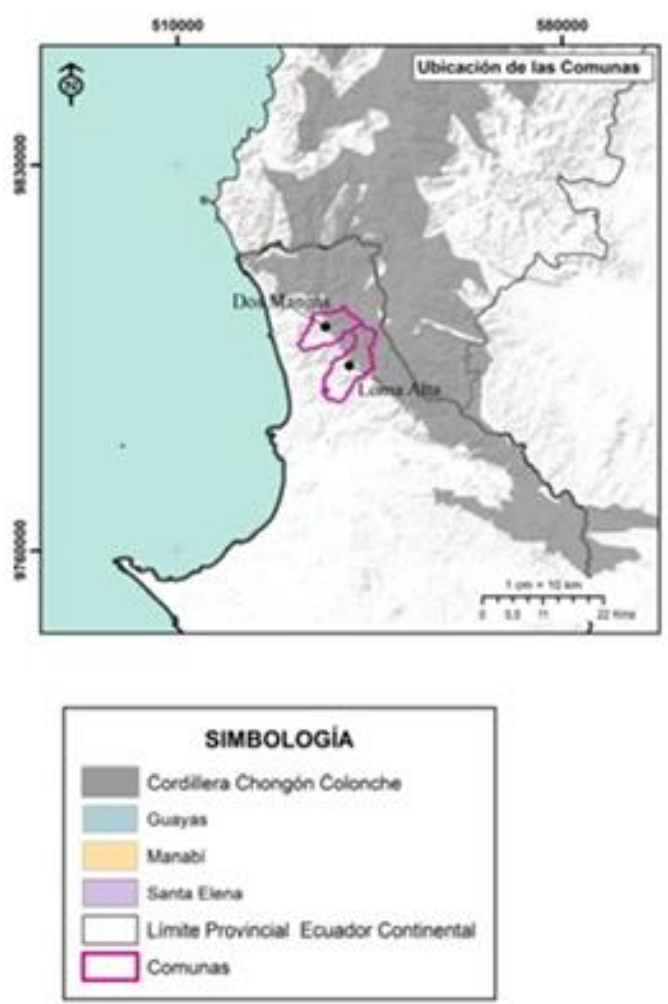

Figura 1. Ubicación del área de estudio dentro de la cordillera Chongón Colonche.

cordillera, la temperatura media oscila entre 24 a $26{ }^{\circ} \mathrm{C}$ (Figura 2) y la precipitación promedio interanual es de $66 \mathrm{~mm} / \mathrm{año}$ con una variabilidad que alcanza los 200 $\mathrm{mm} / \mathrm{mes}$ en época lluviosa o cercanas a cero en la época seca (Figura 2) (GADMCSE, 2014). La temperatura y precipitación cambia durante El Niño - Oscilación Sur (ENOS) que alcanza valores promedio superiores a 35 ${ }^{\circ} \mathrm{C}$ y una precipitación de 2800 mm/año (MAE, 2017).

En Loma Alta, la orografía en los poblados va de 64 m (Loma Alta) hasta 90 m (El Suspiro) y en el área protegida oscila desde $200 \mathrm{~m}$ hasta los $830 \mathrm{~m}$ en el Cerro La Torre (Astudillo et al., 2019). La precipitación promedio anual es de $31.35 \mathrm{~mm}$, con la mayor acumulación en febrero $(101.67 \mathrm{~mm})$ para el período 2005-2015 (Tamariz, 2016). La comuna Dos Mangas presenta temperaturas promedio de $23-24{ }^{\circ} \mathrm{C}$ y una precipitación promedio de $500 \mathrm{~mm} /$ año (MAE, 2017).

Las formaciones vegetales identificadas están asociadas a la Cordillera Costera del Pacífico Ecuatorial (CCPE) que incluyen bosques deciduos ubicados en el piedemonte > 200 msnm; bosque semideciduo localizado en las crestas y laderas $>200$ msnm; bosque siempreverde estacional piemontano situado entre 200 - 400 msnm; y bosque siempreverde estacional montano bajo encontrado a partir de 400 msnm (MAE, 2013), este último localmente conocido como bosque de garúa. La fenología de estos bosques ocurre en dos épocas, en mayo-junio y octubre- noviembre (Astudillo et al., 2015), y está asociada al efecto de los vientos marinos (Bonifaz \& Cornejo, 2004). Asimismo, Bonifaz \& Cornejo (2004) indican que de junio a agosto el $80 \%$ de las especies arbóreas fructifica y de diciembre a marzo (la estación lluviosa) ocurre la floración del $60 \%$ de las especies arbóreas (Figura 3).

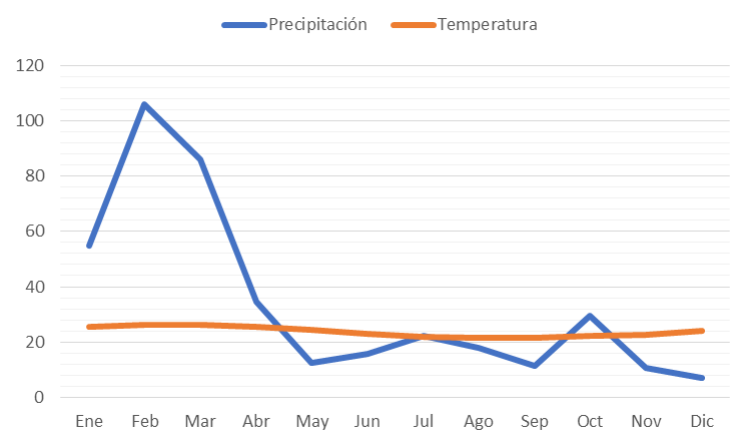

Figura 2. Media mensual de la precipitación (mm) y la temperatura $\left({ }^{\circ} \mathrm{C}\right)$ en estaciones metereológicas ubicadas en la provincia de Santa Elena. La precipitación fue basada en dos estaciones, M619 Manglaralto y M245 Loma Alta. Fuente: GADMCSE, 2014.

La biodiversidad de la CCHC se ha registrado mediante sus taxones encontrando 283 especies botánicas (Astudillo et al., 2019) para Loma Alta; 486 
especies de aves, incluyendo marinas y playeras, de estás 35 son endémicas tumbesinas y 7 endémicas del Chocó (Ágreda, 2012); mientras que, la herpetofauna está compuesta por 20 especies de anfibios y 23 de reptiles, de estas 6 y 5 especies respectivamente son endémicas tumbesinas (Salvatierra et al., 2010).

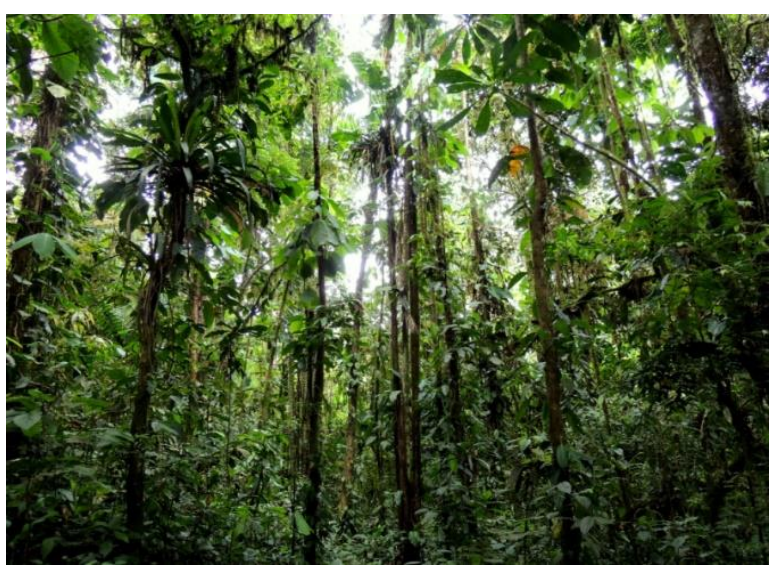

a) Loma Alta

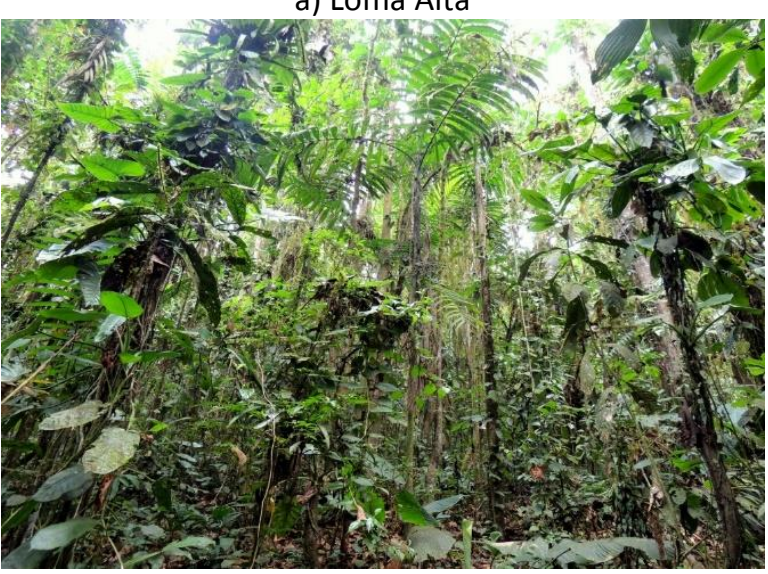

b) Dos Mangas

Figura 3. Vista del bosque de garúa en las dos localidades de la cordillera Chongón Colonche. a) Loma Alta, b) Dos Mangas.

\section{Método de muestreo}

El muestreo vegetal se realizó en el año 2015 , durante la estación húmeda (enero) y estación seca (junio). El estudio de la vegetación siguió el método de Gentry (1995), que consiste en establecer 10 transectos de $50 \mathrm{~m} \times 2 \mathrm{~m}$ con el objetivo de registrar todas las plantas leñosas mayores a $2.5 \mathrm{~cm}$ de Diámetro a la Altura del Pecho (DAP).

La determinación de especies botánicas fue basada en el Catálogo de Plantas Vasculares del Ecuador (Jørgensen \& León Yánez, 1999) y trabajos efectuados en el área (Dodson \& Gentry 1978; Valverde et al., 1979, 1991; Dodson et al., 1985; Dodson \& Gentry, 1991; Bonifaz \& Cornejo 2004, Valverde \& Pérez, 2012; Astudillo et al., 2015); los especímenes recolectados, fueron determinados en el Laboratorio de Ciencias Ambientales de la Universidad Especialidades
Espíritu Santo y posteriormente depositados en el Herbario "Reinaldo Espinosa" de la Universidad Nacional de Loja. El ordenamiento de especies angiospermas se basó en el Sistema de Clasificación del APG IV (APG IV, 2016) y la web Tropicos.org del Missouri Botanical Garden (Tropicos, 2019), así como la determinación de especies endémicas y su estatus de conservación se basó en el Libro rojo de las plantas endémicas del Ecuador (León-Yánez et al., 2011) y los listados de la Unión Internacional para la Conservación de la Naturaleza (UICN, 2019).

Con la finalidad de conocer la estructura vegetal y las especies dominantes se anotó su abundancia en los transectos y se midieron parámetros volumétricos y dasométricos acorde a Aguirre (2013).

Análisis de los resultados

Con los datos volumétricos y dasométricos se obtuvo el Índice de Valor de Importancia (IVI), el cual resalta las especies ecológicas representativas (Curtis, 1959; Soler et al., 2012). Además, se especificaron las clases diamétricas en una escala de $10 \mathrm{~cm}$ y altimétricas en una escala de $5 \mathrm{~m}$.

Se determinó la diversidad alfa $(\alpha)$ de los transectos mediante el índice de diversidad de Shannon-Wienner $(\mathrm{H}$, estima la heterogeneidad de la comunidad considerando su abundancia), el cual se representó en unidades nats por individuo (nats/ind) y el de Simpson (D, que destaca la representatividad de especies raras del resto) (Moreno, 2001; las fórmulas se muestran en la Tabla 2). Se estimó la diversidad beta mediante el índice de Whittaker para conocer la propiedad de reemplazo en las comunidades vegetales (Moreno, 2001; Koleff et al., 2003) según Fórmula 1.

$$
\beta \mathrm{w}=\mathrm{S} / \alpha-1 \text { (Fórmula 1) }
$$

Donde: $\mathrm{S}$ es el número de especies registradas en un conjunto de muestras del paisaje (diversidad gamma) y $\alpha$ es el número promedio de especies en las muestras (Moreno, 2001).

También se consideró la similitud florística entre localidades y se empleó un análisis de clasificación numérica (dendrogramas) a partir de la matriz de abundancia usando el algoritmo UPGMA (Unweighted Pair-Group Average), método que reduce la distorsión en la distancia entre agrupamientos (Oksanen, 2010; Rocha et al., 2010). Para el agrupamiento se empleó el índice de Bray-Curtis porque considera el valor cuantitativo del dato y es asimétrico por no incluir los pares de ausencias en la similitud (Zuur et al., 2007) (Fórmula 2):

$$
\mathrm{CN}=2 \mathrm{jN} /(\mathrm{Na}+\mathrm{Nb})(\text { Fórmula } 2)
$$

Donde: $\mathrm{N}_{\mathrm{a}}$ es el total número de individuos en el sitio $\mathrm{a}, \mathrm{N}_{\mathrm{b}}$ es el total del número de individuos del sitio b, $2 \mathrm{jN}$ es la suma de la menor de las dos abundancias para las especies encontradas en ambos sitios (Newton, 2007).

Adicionalmente, se utilizó un análisis de Ordenación con la técnica de Escalamiento Multidimensional No Métrico (nMDS) para indicar la 


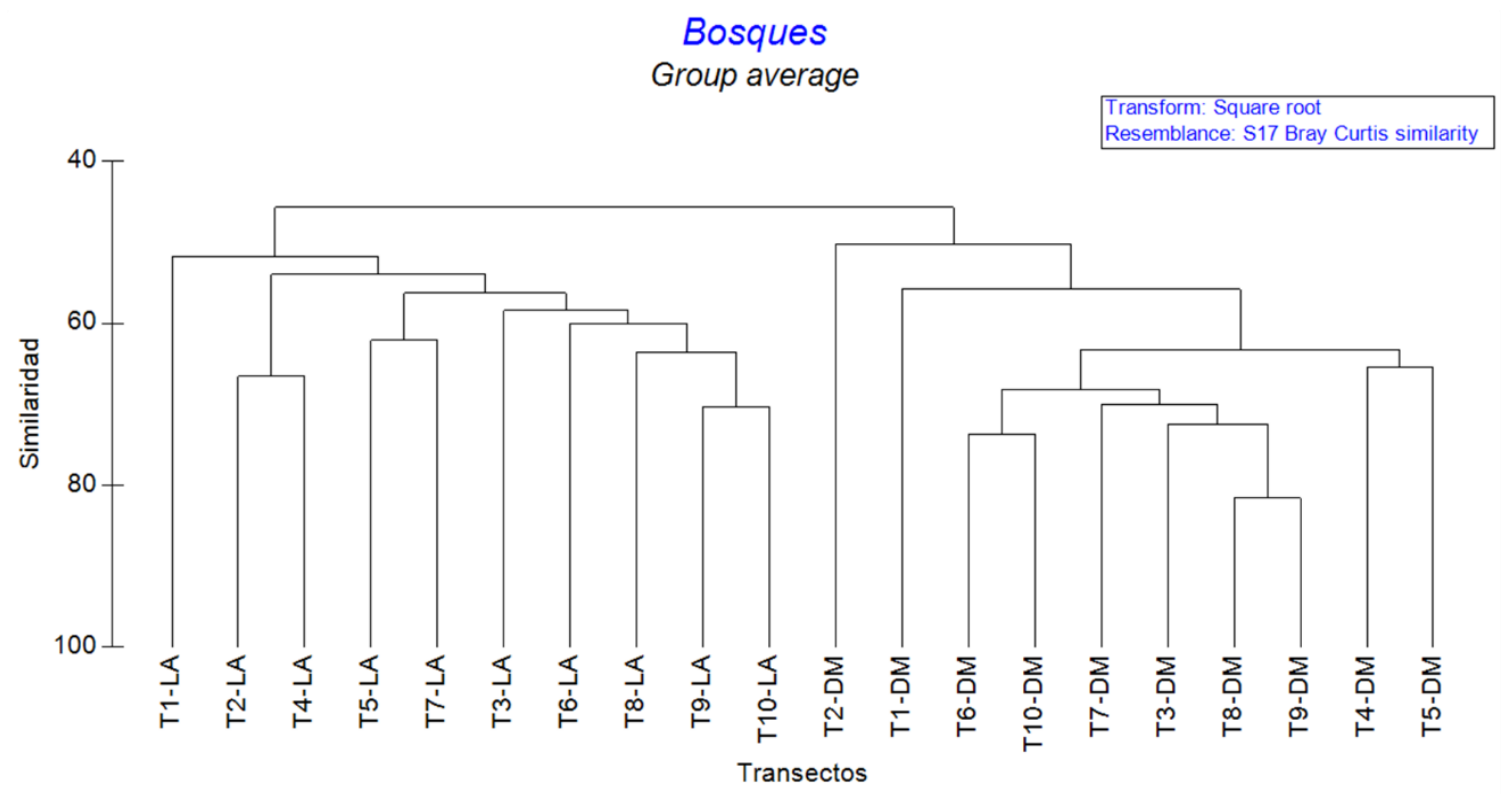

Figura 4. Dendrograma de similaridad de las parcelas entre bosques. LA = Loma Alta; DM = Dos Mangas.

distancia relativa entre sitios en un mapa considerando el nivel de stress (McCune et al., 2002). Para determinar si existían diferencias significativas en la estructura comunitaria entre los bosques de Loma Alta y Dos Mangas se empleó un Análisis Multidimensional No Paramétrico (MDS-Anosim) (Clarke \& Warwick, 2001; Clarke \& Gorley, 2006). Para determinar el aporte de las especies representativas responsables de las diferencias de los grupos vegetales se empleó el método Porcentaje de Similitud (SIMPER) (Kruskal \& Wish, 1978; Clarke, 1993). Todos los análisis se basaron en la medida de distancia Bray-Curtis.

Para los análisis estadísticos se utilizó Microsoft Office Excel 2010 (C Microsoft, bajo licencia), PAST versión 3.16 (Hammer et al., 2001) y PRIMER-E 6.0 (Clarke \& Goley, 2006).

\section{Resultados}

Diversidad y composición florística

En la zona de estudio se registró 48 especies, 46 géneros y 28 familias, las más representativas fueron Arecaceae, Urticaceae, Bignoniaceae, Euphorbiaceae, Lauraceae, Moraceae y Rubiaceae; el género con más especies fue Cecropia (Tabla 1). Según las formas de vida, cuarenta y cuatro especies fueron árboles, dos especies arbustos y dos especies lianas (Tabla 1). Vasconcellea microcarpa, Parinari romeroi y Tanaecium pyramidatum son nuevos registros para la zona de estudio, la primera localizada en Loma Alta y las dos últimas en Dos Mangas.

\section{Diversidad alfa $(\alpha)$}

La riqueza específica fue mayor para Loma Alta con 36 especies y menor para Dos Mangas con 31 especies; mientras tanto la abundancia fue inversa, menor para Loma Alta con 379 individuos y mayor para Dos Mangas con 390 individuos. La diversidad alfa promedio para los bosques de garúa de Loma Alta y Dos Mangas oscila en rangos medios (Tabla 2); aunque, Loma Alta obtuvo el valor promedio más alto ( $\mathrm{H}=2.40$ nats/ind) comparado con el de Dos Mangas ( $\mathrm{H}=2.30$ nats/ind) (Tabla 3 ). La dominancia medida a través del índice de Simpson presentó un patrón inverso a la diversidad con valores en el rango bajo (Tabla 2), siendo los valores promedio 0.13 para Loma Alta y 0.15 para Dos Mangas (Tabla 3).

\section{Diversidad beta $(\beta)$}

El análisis mediante el índice de Whittaker mostró que la tasa de recambio es mayor (1.29) en Loma Alta y menor (1.09) en Dos Mangas. En cuanto a la similaridad de los bosques, en el dendrograma se observó la formación de dos grupos y cada uno representa a una localidad (Figura 4). El análisis de ordenación espacial nMDS mostró, igualmente, dos grupos separados según el bosque (Figura 5); la prueba de ANOSIM mostró diferencias entre estos grupos ( $\mathrm{R}$ $=0.80, \mathrm{p}<0.01)$. El análisis SIMPER mostró que las especies responsables de esta diferencia fueron Chamaedorea linearis "Palmito", Grias peruviana "Aguacatillo" y Catalatola costaricensis "Palo de Pija" que juntas contribuyen al 15\% en disimilitud (Tablas 4 y 5).

\section{Endemismo y estado de conservación}

Se registraron 19 especies endémicas (40\%), de estas 11 son compartidas y 8 a nivel nacional. El endemismo compartido fue extendido para el punto caliente de biodiversidad Tumbes-Chocó-Magdalena (TCM) con tres especies, para el Chocó cinco especies, para la región Tumbesina dos especies y Nectandra subbullata compartida con Venezuela. El endemismo para el Ecuador (Ec), incurrió en la especie Alchornea leptogyna acompañada de siete especies para el occidente ecuatoriano (Tabla 1). Se anotaron 19 especies (40\%) en alguna categoría de amenaza, donde 


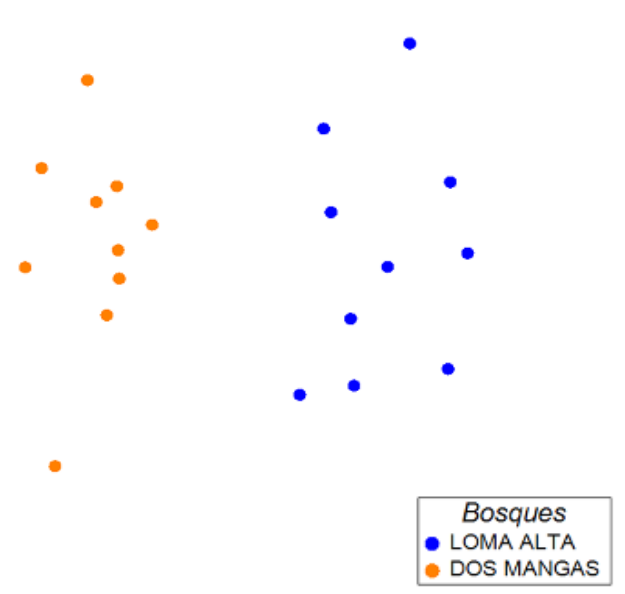

Figura 5. Diagrama de ordenación con nMDS de las comunidades vegetales.

seis están En Peligro (EN), dos como Vulnerable (VU), seis como Casi Amenazada (NT) y cinco como de Preocupación Menor (LC) (Tabla 1). Las especies más importantes para conservar, debido a su estado EN y por ser endémicas fueron Inga carinata "Guaba", Gustavia serrata "Membrillo", Sorocea sarcocarpa "Tillo" y Randia carlosiana "Cañafito".

Estructura

Se diferenciaron tres estratos (e.g., arbóreo, arbustivo y herbáceo) en ambas localidades. En Loma Alta, la estructura estuvo conformada por una vegetación densa (3 790 individuos/ha), donde se detectaron dos estratos, el arbóreo y arbustivo. El estrato arbóreo presenta un dosel discontinuo, donde el $16 \%$ de los individuos alcanzan los $20 \mathrm{~m}$ y el $84 \%$ con alturas promedio de $7.26 \mathrm{~m}$ y un área basal promedio de 0.04, representado por especies como Matisia grandifolia, Randia carlosiana y Calatola costaricensis; mientras tanto, el estrato arbustivo es caracterizado por Psychotria hazenii y árboles regenerantes de la especie Matisia grandifolia. Se registraron las especies más abundantes tales como Matisia grandifolia $(\mathrm{Ar}=26 \%)$, Randia carlosiana $(\mathrm{Ar}$ $=12 \%)$ y Calatola costaricensis $(\mathrm{Ar}=8 \%)$, las cuales alcanzaron el $46 \%$ de la abundancia y se encontraron distribuidas en las diez parcelas; no obstante, sólo la especie Rhodostemonodaphne kunthiana acumuló el $50 \%$ de la dominancia en esta área (Tabla 6). Las cinco especies ecológicamente representativas según el IVI fueron Rhodostemonodaphne kunthiana (18\%), Matisia grandifolia (14\%), Randia carlosiana (7\%), Handroanthus chrysanthus (6\%) y Calatola costaricensis (6\%) (Tabla 6).

En Dos Mangas, la estructura estuvo conformada por una vegetación densa (3900 individuos/ha), donde se identificaron dos estratos bien definidos, el estrato arbóreo y arbustivo. El estrato arbóreo posee un dosel discontinuo, donde el $14 \%$ de los individuos muestra una altura promedio de $18 \mathrm{~m}$, el $45 \%$ con altura promedio de $9.6 \mathrm{~m}$ y el $41 \%$ con alturas promedio de 5 $\mathrm{m}$, así como un área basal promedio de 0.98 con especies representativas tales como Matisia grandifolia, Ficus bullenei y Maquira guianensis. El estrato arbustivo conformado por especies como Psychotria hazenii y la presencia de dos lianas Anemopaegma puberulum y Tanaecium pyramidatum. La abundancia fue determinada por Matisia grandifolia $(\mathrm{Ar}=28 \%)$, Chamaedorea linearis $(\mathrm{Ar}=17 \%) \mathrm{y}$ Psychotria hazenii $(\mathrm{Ar}=6 \%)$ quienes concentraron el $51 \%$ de la abundancia y estuvieron presentes en las diez parcelas, con excepción de la última especie que estuvo en nueve; sin embargo, la especie Ficus bullenei juntó el $45 \%$ de la dominancia (Tabla 7). Las primero cinco especies representativas con mayor IVI fueron Ficus bullenei (16\%), Matisia grandifolia (13\%), Maquira guianensis (9\%), Chamaedorea linearis (8\%) y Nectandra subbullata (8\%) (Tabla 7). Las cinco primeras especies mencionadas representaron entre el $50 \%$ para Loma Alta y 55\% para Dos Mangas del total del IVI para cada área.

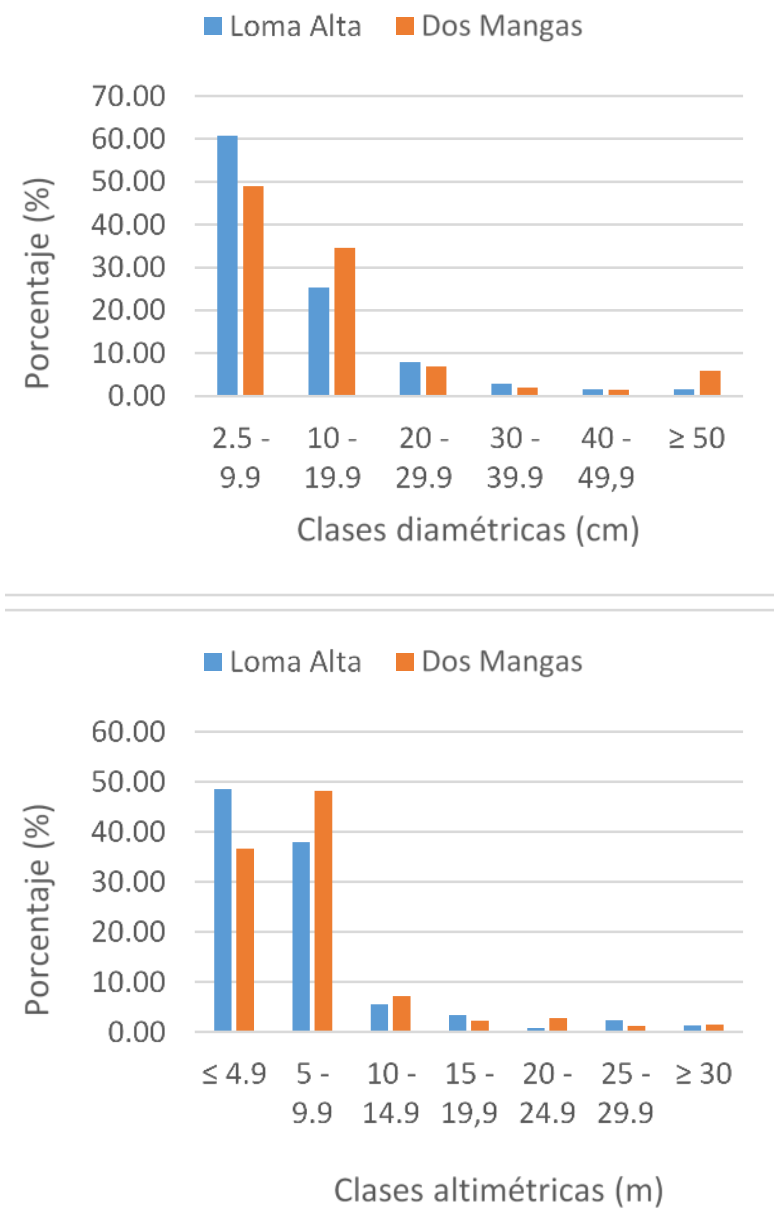

Figura 6. Estructura vegetal del bosque de garúa en la cordillera Chongón Colonche. Se muestran las clases diamétricas (a) y altimétricas (b). 
La estructura horizontal estuvo conformada por seis clases diamétricas, concentrándose más del $50 \%$ en la primera clase $(2.5-9.9 \mathrm{~cm})$ para ambas localidades (Figura 6a); entretanto, la estructura vertical se constituyó por siete clases altimétricas agrupándose el $50 \%$ en la primera clase $(\leq 4.9 \mathrm{~m})$ para Loma Alta y en la segunda clase (5 - $9.9 \mathrm{~m}$ ) para Dos Mangas (Figura $6 b)$.

\section{Discusión}

El aporte de esta investigación contribuye al conocimiento sinecológico en la ecología vegetal de la CCHC estrechando así el vacío de información biológico existente, debido a que Dos Mangas carece de estudios previos y Loma Alta requiere de información actualizada para que ambas comunas fortalezcan la gestión en sus áreas protegidas. En este trabajo, también, se destaca la diversidad de comunidades vegetales que posee el bosque de garúa en ambas localidades y el aporte ecológico de estos bosques como unidad de conservación ya que alberga entre el 40-50\% de especies de los bosques húmedos occidentales del Ecuador y los BES del Pacífico Ecuatorial. El endemismo y estado de conservación de la biodiversidad presente en los bosques de garúa es otro aspecto sobresaliente debido al endemismo local que acorde con Gentry (1986) y Parker \& Carr (1992) está restringido a parches de hábitats en crestas de las montañas que las hacen vulnerables a la extinción.

La composición del bosque de garúa de la $\mathrm{CCHC}$ guarda mayor relación con los bosques húmedos occidentales del Ecuador y contrasta con los BES del Pacífico Ecuatorial. Las familias como Lauraceae, Moraceae y Rubiaceae (Kessler et al., 2012) son representativas de la flora leñosa de los bosques húmedos montanos y estos resultados concuerdan con este trabajo; mientras tanto, las familias Leguminosae, Malvaceae, Boraginaceae y Cactaceae son características de los BES del Pacífico Ecuatorial (Linares-Palomino et al., 2010), donde ellas poseen algunos representantes dentro de esta investigación.

Se realizó una comparación y contraste de la composición florística de los bosques de garúa presentes en la provincia de Santa Elena con otros trabajos a nivel local, nacional y regional, donde se evidencia una mayor similitud florística con los bosques húmedos occidentales del Ecuador y menor con los BES del Pacífico Ecuatorial. El MAE (2013) registró especies diagnósticas para el ecosistema de bosque siempreverde estacional montano bajo de la CCPE (i.e. bosque de garúa) en varias localidades a partir de los $400 \mathrm{msnm}$ hasta las cimas más altas, las cuales coinciden en un 31\% (10 especies y 5 géneros) con esta investigación. Por otra parte, en Loma Alta se realizó un estudio previo por Bonifaz \& Cornejo (2004), quienes generaron un listado botánico que concuerda en un $85 \%$ (40 especies y 1 género) y Jadán et al. (2014) señalaron especies indicadoras para el
Bosque Protector Chongón Colonche (BPCHC) correspondiendo un $33 \%$ (9 especies y 7 géneros) con esta investigación.

Desde la perspectiva de bosques occidentales, Parker \& Carr (1992) recopilaron resultados en ocho diferentes localidades de la región litoral enfocándose en los tres tipos de vegetación que Dodson \& Gentry (1991) describieron para el occidente de Ecuador. Basado en el listado botánico de Parker \& Carr (1992), el bosque de garúa del Parque Nacional Machalilla (PNM) guarda la más alta relación florística $(58 \%, 7$ especies y 21 géneros) con las especies de esta investigación comparado con los bosques húmedos del Cerro Mutiles (48\%, 6 especies y 17 géneros) o de Cabeceras de Bilsa (46\%, 6 especies y 16 géneros), ambas en Esmeraldas, o con el bosque de garúa de Manta Real (44\%, 4 especies y 17 géneros), en Azuay.

En cambio, si esto lo contrastamos con el listado del bosque seco ( $31 \%, 5$ especies y 10 géneros) del PNM o del Bosque Protector Cerro Blanco (29\%, 2 especies y 12 géneros), ambos ubicados en la $\mathrm{CCHC}$, observamos una menor similitud florística. Algo similar ocurre con el trabajo de Aguirre \& Delgado (2005) para los bosques secos de la provincia de Loja en el sur del país o Aguirre et al. (2006b) para los BES del Pacífico Ecuatorial, los cuales contienen el 19\% (1 especies y 8 géneros) y $40 \%$ (6 especies y 13 géneros) de especies reportadas para el bosque de garúa; así como la investigación de Linares-Palomino, 2004 representó el 23\% (1 especie y 9 géneros) para los BES del Perú.

La composición y diversidad florística del bosque de garúa se debe a la presencia de la cordillera sensu stricto y a la precipitación horizontal que son factores que singularizan a estos bosques. Las cadenas montañosas tienen un rol secundario en la determinación de los factores climáticos y provocan el efecto de lluvias orográficas (Ricklefs et al., 2014), las mismas que transforman las condiciones climáticas áridas características de la región litoral ecuatoriana cambiando así los patrones biológicos y albergando una vegetación húmeda. Acorde a Bonifaz \& Cornejo (2004), la CCHC modifica la composición florística que es similar a los bosques húmedos de las estribaciones de la cordillera de los Andes (e.g., Río Palenque a $2980 \mathrm{msnm}$ ) y que Gentry $(1982,1995)$ encontró remarcable debido a que dos tercios de sus muestras vegetales tomadas en el PNM fueron similares a las de Río Palenque y no a Jauneche, que es un área geográficamente cercana con una precipitación semejante (Parker \& Carr, 1992).

La diversidad $\alpha$ estudiada en los bosques de la CCHC y en los BES del Pacífico Ecuatorial muestran patrones similares. Estudios previos de diversidad alfa reportaron valores medios similares para el BPCHC (2.22-2.65 nats/ind; Jadán et al., 2014) y para los bosques secos de la provincia de Loja en Ecuador (2.45 a 2.75 nats/ind; Aguirre et al., 2013a). Otras investigaciones en los BES de Perú observaron valores 
similares para Amotape y Tumbes (1.17 - 3.03 nats/ind; Leal-Pinedo \& Linares-Palomino, 2005) y para Jaén (2.9 a 4.1 nats/ind; Marcelo-Peña et al., 2007). Desafortunadamente, no existen estudios previos de diversidad $\alpha$ para los bosques de garúa de la costa ecuatoriana o de la región del Chocó ecuatoriano para comparar.

Sin embargo, estas diferencias en los resultados de riqueza y abundancia estimada mediante índices se podrían explicar por la injerencia de factores ecológicos que generan patrones en la distribución de la diversidad. La altitud, disponibilidad de agua y la geología del área son factores ecológicos que afectan la diversidad y composición vegetal, especialmente en plantas leñosas que tienen una fuerte relación con la precipitación y estacionalidad en escalas geográficas y locales (Kessler et al., 2012). A escala regional la riqueza específica y la distribución de especies vegetales leñosas de los BES del Ecuador y Perú obedecen un patrón conforme al gradiente altitudinal, donde Ecuador muestra un incremento de valores hacia las altitudes medias (colinas) y se reduce hacia las altitudes bajas (tierras bajas) o altitudes altas (montañas); entretanto que, Perú tiene un patrón de aumento en tierras bajas costeras hacia la zona premontana y decrece en las montañas (LinaresPalomino et al., 2010). A escala local, Gentry, en su estudio en el PNM, destacó los cambios entre las diferentes comunidades vegetales dependiendo del gradiente altitudinal (Parker \& Carr, 1992); mientras tanto, Astudillo-Sánchez et al. (2019) manifestaron que existe una tendencia de incremento de la diversidad alfa siguiendo un gradiente altitudinal, pero su trabajo no fue conclusivo debido a que se limitó a un rango entre 100 a 500 msnm en la comuna Loma Alta.

La diversidad $\beta$ medida desde la similitud florística y mediante los análisis de ordenación nMDSANOSIM-SIMPER definió dos grupos florísticos y esto podría atribuirse a las condiciones climáticas y la configuración espacial que padecen ambos bosques con respecto a la exposición al viento, lo cual genera microclimas en el área de estudio. A pesar de que ambas localidades son áreas contiguas geográficamente que se ubican en la vertiente occidental de la CCHC y la muestra fue tomada en un rango altitudinal de 500 msnm, la comuna Dos Mangas presenta un mayor régimen de precipitación debido a que está más cercana al océano y por ello recibe mayor humedad horizontal (i.e. garúa) que la comuna Loma Alta que está en tierra adentro de la misma vertiente con menor precipitación y un clima seco.

El endemismo y conservación de la $\mathrm{CCHC}$ es otro aspecto para resaltar ya que acorde a Sierra (2018), la región litoral alberga casi el doble de especies endémicas por superficie que la región amazónica y según Cornejo (2018), los bosques húmedos concentran el endemismo de la región occidental del Ecuador quienes estan representados por las familias
Orchidaceae, Araceae, Gesneriaceae, Bromeliaceae y Ericaceae; así como la familia Capparaceae como endémica. Las especies Capparidastrum bonifazianum, Inga carinata, Gustavia serrata, Sorocea sarcocarpa y Randia carlosiana son especies endémicas para los bosques del litoral y las estribaciones occidentales, las cuales están amenazadas por la tala selectiva que continúa afectando estas dos áreas protegidas ya que durante el desarrollo de esta investigación hemos sido testigos de esta actividad. Adicionalmente, a escala regional, el endemismo del Pacífico Ecuatorial sigue un patrón de aumento desde elevaciones bajas hacia medias y mermaron en elevaciones altas (LinaresPalomino et al., 2010).

La problemática de la conservación de los bosques secos se refleja en los datos de la estructura del bosque para ambas localidades, donde se observa que el $50 \%$ son individuos jóvenes con menos de $10 \mathrm{~cm}$ de DAP y menos del $10 \%$ de los individuos se agrupan en la categoría diamétrica $\geq 30 \mathrm{~cm}$ de DAP. La gráfica del análisis estructural muestra una $\mathbf{J}$ invertida típica de bosques nativos, maduros, estables con regeneración favorable (Leal-Pinedo \& Linares-Palomino, 2005; Aguirre, 2013); no obstante, al concentrar los individuos en la primera clase diamétrica se pueden relacionar estos resultados con bosques secundarios jóvenes en procesos de recuperación que han sido afectados por la tala selectiva (Yépez et al., 2009; Aguirre et al., 2013b; Muñoz et al., 2014). Asimismo, el estado de conservación de los bosques del litoral ha sido explicado por Cornejo (2018) quién urge la conservación privada debido a que las áreas protegidas del SNAP siguen afectadas y en los remanentes de bosques aún se siguen descubriendo nuevas especies, muchas de ellas endémicas.

La ausencia de la especie maderable Handroanthus chrysanthus "Guayacán" en Dos Mangas y la baja abundancia en Loma Alta son otro ejemplo de la presión antrópica a la que están expuestos ambas áreas protegidas, ya que esta especie es representativa del bosque seco y tiene un alto uso consuntivo maderable en el Sur de Ecuador (Aguirre \& Delgado, 2005; Aguirre et al., 2006a, 2006b, 2013b; Muñoz et al., 2014) y para el Norte de Perú (Linares-Palomino et al., 2010)

\section{Conclusiones}

A escala local, la cordillera Chongón Colonche presenta condiciones climáticas y orográficas que permite cambios en la composición florística presente y los bosques de garúa sobresalen por su aporte a la diversidad taxonómica distinguiéndose en ellos familias de bosque húmedo en un área caracterizada por un clima xérico. De igual manera, la cordillera contribuye a la existencia de un endemismo local para el occidente ecuatoriano albergando especies como Inga carinata, Gustavia serrata y Capparidastrum bonifazianum que están catalogadas como endémicas y 
En Peligro. A escala regional, los bosques estacionalmente secos necesitan de una estrategia de conservación enfocada en los grupos florísticos vulnerables que permita la planificación y gestión eficiente de su biodiversidad, ya que comparten los mismos problemas socio-ambientales que soslaya los servicios ecosistémicos para el beneficio humano e históricamente han sido aprovechados. La conservación y manejo eficiente es urgente y necesario a escala local en estas dos áreas protegidas que geográficamente son contiguas, pero florísticamente son disimiles en un $54 \%$ y que tienen una alta tasa de recambio vegetal.

\section{Agradecimientos}

Agradecemos al Centro de Investigaciones de la Universidad de Especialidades Espíritu Santo (UEES) por el financiamiento para la ejecución de este proyecto UEES-2014-ART-002 y a todos los estudiantes que participaron en el trabajo de campo. Al Ministerio del Ambiente del Ecuador (MAE), a través de su Dirección Provincial de Santa Elena, por el permiso de investigación correspondiente $\mathrm{N}^{\circ}$ 017-15 IC-FAUDPSE-MA y al Dr. Zhofre Aguirre, director del Herbario Nacional de Loja, por la recepción de las muestras botánicas. Al cabildo de la comuna Loma Alta y Dos Mangas, representados por sus presidentes, Abg. Gustavo de la A y Sr. Ángel Merchán, y a los guardabosques. Un especial agradecimiento a los revisores anónimos y editores que han enriquecido esta versión final.

\section{Literatura citada}

Ágreda A. 2012. Check list de las aves de la Cordillera Chongón - Colonche y áreas protegidas en la provincia del Guayas, Santa Elena y Manabí. The Nature Conservancy; Aves y Conservación (Birdlife International en Ecuador). Guayaquil / Ecuador.

Aguirre Z. \& Delgado T. 2005. Vegetación de los bosques secos de Cerro Negro-Cazaderos, occidente de la provincia de Loja. En: Vázquez M.A., Freile J.F. \& Suárez L. (eds.) Biodiversidad en los bosques secos de la zona de Cerro Negro-Cazaderos, occidente de la provincia de Loja: un reporte de las evaluaciones ecológicas y socioeconómicas rápidas. 9-24. EcoCiencia, MAE y Proyecto Bosque Seco, Quito.

Aguirre Z. 2013. Guía de Métodos para Medir la Biodiversidad. Universidad Nacional de Loja. Loja, Ecuador.

Aguirre Z., Betancourt Y., Geada G. \& Jasen H. 2013a. Composición florística, estructura de los bosques secos y su gestión para el desarrollo de la provincia de Loja, Ecuador. Avances, 15(2): 144-155.

Aguirre Z., Geada G. \& Betancourt Y. 2013b. Regeneración natural en los bosques secos de la provincia de Loja y utilidad para el manejo local. Revista Cedamaz, 3(1): 5465.

Aguirre Z., Kvist L.P. \& Sánchez O. 2006a. Bosques secos en Ecuador y su diversidad. Botánica Económica de los Andes Centrales: 162-187.
Aguirre Z., Linares-Palomino R. \& Kvist L.P. 2006 b. Especies leñosas y formaciones vegetales en los bosques estacionalmente secos de Ecuador y Perú. Arnaldoa, 13(2): 324-350.

APG IV (Angiosperm Phylogeny Group IV). 2016. An update of the Angiosperm Phylogeny Group classification for the orders and families of flowering plants: APG IV. Botanical Journal of the Linnean Society, 181: 1-20.

Astudillo E., Pérez J. \& Fabara M. 2015. Árboles y arbustos: una relación con la avifauna. Samborondón: Universidad Espíritu Santo - Ecuador.

Astudillo-Sánchez E., Pérez J., Troccoli L. \& Aponte H. 2019. Composición, estructura y diversidad vegetal de la Reserva Ecológica Comunal Loma Alta, Santa Elena, Ecuador. Revista Mexicana de Biodiversidad, 90(1): 125. DOI: $10.22201 / \mathrm{ib} .20078706 \mathrm{e} .2019 .90 .2871$.

Banda K., Delgado-Salinas A., Dexter K.G., LinaresPalomino R., Oliveira-Filho A., Prado D., Weintritt J., et al. 2016. Plant diversity patterns in neotropical dry forests and their conservation implications. Science, 353(6306): 1383-1387. DOI: $10.1126 /$ science.aaf5080.

Bonifaz C. \& Cornejo X. 2004. Flora del Bosque de Garúa (árboles y epifitas) de la Comuna Loma Alta, cordillera Chongón Colonche, provincia del Guayas, Ecuador. USA: Missouri Botanical Garden Press.

Campo A.M. \& Duval V.S. 2014. Diversidad y valor de importancia para la conservación de la vegetación natural. Parque Nacional Lihué Calel (Argentina). Anales de Geografía de la Universidad Complutense 34 (2): 25 42.

CEPF (Critical Ecosystem Partnership Fund). 2005. Corredor de Conservación Chocó-Manabí Ecorregión Terrestre Prioritaria del Chocó-Darién-Ecuador Occidental (Hotspot).

https://www.cepf.net/sites/default/files/final.spanish.cho co-darien-western-ecuador.choco_.ep_.pdf.

Clarke K.R. \& Gorley R.N. 2006. PRIMER v6. User Manual/ Tutorial. PRIMER-E. Plymouth.

Clarke K.R. \& Warwick R.M. 2001. Change in marine communities: An approach to statistical analyses and interpretation. 2nd ed. Primer-E. Plymouth, UK.

Clarke K.R. 1993. Non-parametric multivariate analyses of changes in community structure. Australian Journal of Ecology 18: 117-143.

Cornejo X. 2018. Libro rojo de las plantas endémicas del Ecuador: Endemismo en la región litoral. Consultado el 09 de junio de 2019 de: https://bioweb.bio/floraweb/librorojo/litoral/.

Cuesta F., Peralvo M., Baquero F., Bustamante M., Merino A., Muriel P., Freile J. \& Torres O. (eds.). 2013. Identificación de vacíos y prioridades de conservación en el Ecuador Continental. Consorcio para el Desarrollo Sostenible de la Ecorregión Andina. Quito.

Cuesta F., Peralvo M., Merino-Viteri A., Bustamante M., Baquero F., Freile J.F., et al. 2017. Priority areas for biodiversity conservation in mainland Ecuador. Neotropical Biodiversity, 3(1): 93-106.

Curtis J. 1959. The vegetation of Wisconsin. An ordination of plant communities. Univ. of Wisconsin Press. Madison. EUA

Dodson C.H. \& Gentry A.H. 1978. Flora of the Río Palenque Science Center, Los Ríos, Ecuador. Selbyana 4. 
Dodson C.H. \& Gentry A.H. 1991. Biological extinction in western Ecuador. Annals of the Missouri Botanical Garden: 273-295.

Dodson C.H., Gentry A.H. \& Valverde F.M. 1985. Flora de Jauneche. Banco Central del Ecuador. Quito, Ecuador.

Eduardo-Palomino F., Chuquillanqui H., Najarro P. \& Linares-Palomino R. 2017. Contribución a la flora vascular y vegetación de los valles secos interandinos de los ríos Torobamba (Ayacucho) y Pampas (Apurímac), sur del Perú. Ecología Aplicada, 16(2): 115-125. DOI: http://dx.doi.org/10.21704/rea.v16i2.1015.

Eguiguren-Velepucha P.A., Chamba J.A.M., Aguirre N.A., Ojeda-Luna T.L., Samaniego-Rojas N.S., Furniss M.J., Howe C. \& Aguirre Z.H. 2016. Tropical ecosystems vulnerability to climate change in southern Ecuador. Tropical Conservation Science, 9(4): 1-17. DOI: $10.1177 / 1940082916668007$.

Escribano-Avila G. 2016. El bosque seco neotropical de la provincia ecuatoriana: un pequeño gran desconocido. Revista Ecosistemas, 25(2): 1-4.

Escribano-Avila G., Cervera L., Ordóñez-Delgado L., JaraGuerrero A., Amador L., Paladines B., Briceño J., ParésJiménez V., Lizcano D.J., Duncan D.H. \& Espinosa C.I. 2017. Biodiversity patterns and ecological processes in Neotropical dry forest: the need to connect research and management for long-term conservation. Neotropical Biodiversity, 3(1): 107-116. DOI: https://doi.org/10.1080/23766808.2017.1298495.

Espinosa C.I., De la Cruz M., Luzuriaga A.L. \& Escudero A. 2012. Bosques tropicales secos de la región Pacífico Ecuatorial: diversidad, estructura, funcionamiento e implicaciones para la conservación. Revista Ecosistemas 21: 1-2.

GADMCSE (Gobierno Autónomo Descentralizado Municipal - Cantón Santa Elena / Ecuador). 2014. Plan de Desarrollo y Ordenamiento Territorial - Cantón Santa Elena 2014-2019.

García-Villacorta R. 2009. Diversidad, composición y estructura de un hábitat altamente amenazado: los bosques estacionalmente secos de Tarapoto, Perú. Revista Peruana de Biología, 16(1): 81-92. DOI: https://doi.org/10.15381/rpb.v16i1.177.

Gentry A.H. 1986. Species richness and floristic composition of Chocó region plant communities. Caldasia, 15: 71-91.

Gentry A.H. 1995. Diversity and floristic composition of neotropical dry forest. In: Bullock SH, Mooney HA, Medina E (eds) Seasonally dry tropical forest. Cambirdge University Press, Cambridge.

Hammer O., Harper D.A.T. \& Ryan P.D. 2001. PAST: Paleontological Statistics software package for education and data analysis. Paleontologia Electronica 4 (1): 9.

Jadán O., Veintimilla D., Ponce E., González M., Waise H. \& Aguirre Z. 2014. Identificación y caracterización florística de bosques naturales en el Bosque Protector Chongón Colonche, Ecuador. Revista Bosques Latitud Cero, 4: 7-14.

Jørgensen P.M. \& León-Yánez S. 1999. Catálogo de las plantas vasculares del Ecuador. Monographs in systematic botany from the Missouri Botanical Garden 75: 1-1181.
Kessler M., Grytnes J.A., Halloy S.R., Kluge J., Krömer T., León B., Macía M.J. \& Young K.R. 2012. Gradientes de diversidad vegetal: Patrones y procesos locales. En: Herzog S.K., Martinez R., Jørgensen P.M. \& Tiessen H. (eds.) Cambio climático y biodiversidad en los Andes Tropicales. 235-253. Instituto Interamericano para la Investigación del Cambio Global (IAI); Comité Científico sobre Problemas del Medio Ambiente (SCOPE) / UNESCO. http://www.iai.int/admin/site/sites/default/files/libro_co mpleto.pdf.

Koleff P., Gaston K. \& Lennon J. 2003. Measuring beta diversity for presence-absence data. Journal of Animal Ecology, 72(3): 367-382.

Kruskal J.B. \& Wish M. 1978. Quantitative Applications in the Social Sciences: Multidimensional scaling. Thousand Oaks, CA: SAGE Publications Ltd.

Leal-Pinedo J.M. \& Linares-Palomino R. 2005. Los bosques secos de la Reserva de Biosfera del Noroeste (Perú): Diversidad arbórea y estado de conservación. Caldasia, 27(2): 195-211.

León-Yánez S., Valencia R., Pitman N., Endara L., Ulloa C. \& Navarrete H. 2011. Libro rojo de las plantas endémicas del Ecuador, segunda edición. Publicaciones del Herbario QCA, Pontificia Universidad Católica del Ecuador. Quito, Ecuador.

Linares-Palomino R. 2004. Los bosques tropicales estacionalmente secos: II. Fitogeografía y composición florística. Arnaldoa, 11 (1): 103-138.

Linares-Palomino R., Kvist L.P., Aguirre-Mendoza Z. \& Gonzales-Inca C. 2010. Diversity and endemism of woody plant species in the Equatorial Pacific seasonally dry forests. Biodiversity and Conservation, 19:169-185.

MAE (Ministerio del Ambiente de Ecuador). 2012. Socio Bosque. Línea Base de Deforestación del Ecuador Continental. Quito / Ecuador.

MAE (Ministerio del Ambiente de Ecuador). 2013. Sistema de Clasificación de los Ecosistemas del Ecuador Continental. Subsecretaría de Patrimonio Natural. Quito.

MAE (Ministerio del Ambiente del Ecuador). 2017. Informe del taller participativo "Análisis de vulnerabilidad local al cambio climático del sector ganadero en las zonas de implementación del proyecto MGCI en la provincia de Santa Elena".

Marcelo-Peña J.L., Reynel-Rodríguez C., Zevallos-Pollito P., Bulnes-Soriano F. \& Pérez-Ojeda del Arco A. 2007. Diversidad, composición florística y endemismos en los bosques estacionalmente secos alterados del distrito de Jaén, Perú. Ecología aplicada, 6(1-2): 9-22.

Marchese C. 2015. Biodiversity hotspots: A shortcut for a more complicated concept. Global Ecology and Conservation, 3: 297-309.

McCune B., Grace J.B. \& Urban D.L. 2002. Analysis of ecological communities. MJM Software Desig.

Mittermeier R.A., Turner W.R., Larsen F.W., Brooks T.M. \& Gascon C. 2011. Global biodiversity conservation: the critical role of hotspots. In: Biodiversity hotspots. 3-22. Springer. Heidelberg, Berlin.

Moreno C.E. 2001. Métodos para medir la biodiversidad. M\&T- Manuales y Tesis SEA, Vol 1. Zaragoza.

Muñoz J., Erazo S. \& Armijos D. 2014. Composición florística y estructura del bosque seco de la quinta experimental "El Chilco" en el suroccidente del Ecuador. Cedamaz, 4(1): 53-61. 
Newton A. 2007. Forest ecology and conservation: a handbook of techniques. Oxford University Press on Demand.

Oksanen J. 2010. Multivariate analysis of ecological communities in R: vegan tutorial.

Palacios W.A. \& Jaramillo N. 2016. Árboles amenazados del Chocó ecuatoriano. ACI Avances en Ciencias e Ingenierías, 8(14): 51-60.

Paladines R. 2003. Propuesta de conservación del Bosque seco en el Sur de Ecuador. Lyonia, 4(2): 183-186.

Parker T.A. \& Carr J.L. (eds). 1992. Status of the forest remnants in the Cordillera de la Cosa and Adjacent areas of Southwestern Ecuador. Rapid Assessment Program Working Paper 2. Conservation International, Washington D.C.

Pennington R.T., Lavin M. \& Oliveira-Filho A. 2009. Woody plant diversity, evolution, and ecology in the tropics: perspectives from seasonally dry tropical forests. Annual Review of Ecology, Evolution and Systematics, 40: 437457.

Pennington R.T., Lavin M., Prado D.E., Pendry C.A., Pell S.K. \& Butterworth C.A. 2004. Historical climate change and speciation: neotropical seasonally dry forest plants show patterns of both Tertiary and Quaternary diversification. Philosophical Transactions of the Royal Society of London. Series B: Biological Sciences, 359(1443): 515-538.

Portillo-Quintero C., Sanchez-Azofeifa A., Calvo-Alvarado J., Quesada M. \& do Espirito Santo M.M. 2015. The role of tropical dry forests for biodiversity, carbon and water conservation in the neotropics: lessons learned and opportunities for its sustainable management. Regional Environmental Change, 15(6): 1039-1049.

Ricklefs R.E., Relyea R. \& Richter C. 2014. Ecology: The economy of nature.W.H. Freeman and Cia. Seventh Edition, Canadian edition.

Rocha A., Ramírez N. \& González M. 2010. Riqueza y diversidad de árboles del bosque tropical caducifolio en la Depresión Central de Chiapas. Boletín de la Sociedad Botánica de México 87:89-103.

Salvatierra B., Ortega J. \& Amador L. 2010. Evaluación Ecológica Rápida de la Herpetofauna en la cordillera Chongón - Colonche, Ecuador. Investigación Tecnología e Innovación, s/vol/n: 52-74. Universidad de Guayaquil.

Sierra R. 2018. Libro rojo de las plantas endémicas del Ecuador: Una aproximación al estado de conservación de los ecosistemas terrestres del Ecuador continental. Consultado el 09 de junio de 2019 de: https://bioweb.bio/floraweb/librorojo/litoral/.
Soler E., Berroterán P., Gil J. \& Acosta R. 2012. Índice valor de importancia, diversidad y similaridad florística de especies leñosas en tres ecosistemas de los llanos centrales de Venezuela. Agronomía Tropical, 62(1-4): 025-038.

Tamariz I. 2016. Propuesta de un Sistema de Aprovechamiento Pluvial para una Escuela de la Comuna Loma Alta. Tesis de Grado. Escuela de Ciencias Ambientales / Facultad de Artes Liberales y Ciencias de la Educación / Universidad de Especialidades Espíritu Santo. Samborondón / Ecuador.

Tropicos. 2019. Tropicos.org. Missouri Botanical Garden. Disponible en: http://www.tropicos.org.

UICN (Unión Internacional para la Conservación de la Naturaleza). 2019. IUCN Red List of Threatned Species. Version 2018-2. Disponible en: http://www.iucnredlist.org/.

Valverde F.M. \& Pérez J. 2012. La biodiversidad vegetal, como capital natural de la sostenibilidad en la Costa Ecuatoriana. Programa Editorial de la M.I. Municipalidad de Santiago de Guayaquil.

Valverde F.M., García C. \& Rodríguez G. 1991. Estado actual de la vegetación natural de la Cordillera de Chongón Colonche. Instituto de Investigaciones de Recursos Naturales / Facultad de Ciencias Naturales / Universidad de Guayaquil.

Valverde F.M., Rodríguez G. \& García C. 1979. Cubierta vegetal de la península de Santa Elena. I parte: Península de Santa Elena. Universidad de Guayaquil. Guayaquil / Ecuador.

Vázquez M.A., Freile J.F. \& Suárez L. (eds). 2005. Biodiversidad en los bosques secos de la zona de Cerro Negro-Cazaderos, occidente de la provincia de Loja: un reporte de las evaluaciones ecológicas y socioeconómicas rápidas. EcoCiencia, MAE \& Proyecto Bosque Seco. Quito.

Yepes A.P., del Valle J.I., Jaramillo S.L. \& Orrego S.A. 2010. Recuperación estructural en bosques sucesionales andinos de Porce (Antioquia, Colombia). Revista de Biología tropical, 58(1): 427-445.

Zuur A.F., Ieno E.N. \& Smith G.M. 2007. Analysing Ecological Data. En: Gail M., Krickeberg K., Samet J., Tsiatis A. \& Wong W. (eds). Statistic for Biology and Health. 163-179. Springer. NY. 


\section{Apéndice}

Tablas.

Tabla 1. Listado de las especies vegetales encontradas en Loma Alta y Dos Mangas. Se indica la familia, especie, estrato, si presenta endemismo se indica la región, la categoría de conservación $($ En Peligro $=$ EN, Vulnerables $=$ VU, Casi Amenazada $=$ NT y Preocupación Menor $=$ LC) y el área donde se ha reportado $($ Loma Alta $=$ LA, Dos Mangas $=$ DM o ambas).

\begin{tabular}{|c|c|c|c|c|c|}
\hline FAMILIAS & NOMBRE ESPECIE & ESTRATO & ENDEMISMO & 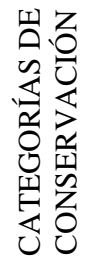 & ÁREA \\
\hline Arecaceae & Bactris setulosa H. Karsten & Arbóreo & & NT & LA \\
\hline Arecaceae & Chamaedorea linearis (Ruiz \& Pav.) Mart. & Arbóreo & & NT & Ambas \\
\hline Arecaceae & Geonoma undata Klotzsch & Arboreo & & NT & DM \\
\hline Arecaceae & Phytelephas aequatorialis Spruce & Arbóreo & Occidente Ec & NT & DM \\
\hline Anacardiaceae & Mauria heterophylla Kunth & Arbóreo & Tumbesina & & $\mathrm{DM}$ \\
\hline Annonaceae & Annona oligocarpa R.E. Fr. & Arbóreo & & $\mathrm{EN}$ & LA \\
\hline Apocynaceae & Tabernaemontana amygdalifolia Jacq. & Arbóreo & & $\mathrm{LC}$ & LA \\
\hline Apocynaceae & Rauvolfia tetraphylla $\mathrm{L}$. & Arbóreo & & & $\mathrm{DM}$ \\
\hline Araliaceae & Dendropanax macrocarpus Cuatrec. & Arbóreo & Chocó & & Ambas \\
\hline Bignoniaceae & Anemopaegma puberulum (Seibert) Miranda & Liana & & & DM \\
\hline Bignoniaceae & Handroanthus chrysanthus (Jacq.) S. O. Grose & Arbóreo & & & LA \\
\hline Bignoniaceae & Tanaecium pyramidatum (Rich.) L.G. Lohmann & Liana & & & $\mathrm{DM}$ \\
\hline Boraginaceae & Cordia collococca $L$ & Arbóreo & & $\mathrm{LC}$ & Ambas \\
\hline Capparaceae & Capparidastrum bonifazianum Cornejo \& Iltis & Arbóreo & Occidente Ec & NT & LA \\
\hline Caricaceae & Vasconcellea microcarpa (Jacq.) A. DC. & Arbustivo & & & LA \\
\hline Chrysobalanaceae & Parinari romeroi Prance & Arbóreo & & VU & $\mathrm{DM}$ \\
\hline Clusiaceae & Chrysochlamys dependens Planch. \& Triana & Arbóreo & TCM & & Ambas \\
\hline Clusiaceae & Garcinia madruno (Kunth) B. Hammel & Arbóreo & & & LA \\
\hline Euphorbiaceae & Acalypha cuneata Poepp. & Arbóreo & & & Ambas \\
\hline Euphorbiaceae & Alchornea leptogyna Diels & Arboreo & $\mathrm{Ec}$ & NT & LA \\
\hline Euphorbiaceae & Tetrorchidium andinum Müll. Arg. & Arbóreo & & & Ambas \\
\hline Fabaceae & Dussia lehmannii Harm & Arbóreo & Chocó & & Ambas \\
\hline Fabaceae & Inga carinata T.D. Penn. & Arbóreo & Occidente Ec & $\mathrm{EN}$ & Ambas \\
\hline Lamiaceae & Aegiphila alba Moldenke & Arbóreo & Chocó & & Ambas \\
\hline Lauraceae & Beilschmiedia alloiophylla (Rusby) Kosterm. & Arbóreo & & & Ambas \\
\hline Lauraceae & Nectandra subbullata Rohwer & Arbóreo & Ec-Ve & VU & Ambas \\
\hline Lauraceae & Rhodostemonodaphne kunthiana (Nees) Rohwer & Arbóreo & & & LA \\
\hline Lecythidaceae & Grias peruviana Miers & Arbóreo & Tumbesina & & DM \\
\hline Lecythidaceae & Gustavia serrata S.A Mori & Arbóreo & Occidente Ec & $\mathrm{EN}$ & LA \\
\hline Malvaceae & Matisia grandifolia Little & Arbóreo & Chocó & $\mathrm{EN}$ & Ambas \\
\hline Meliaceae & Carapa guianensis Aubl. & Arbóreo & & $\mathrm{LC}$ & DM \\
\hline Metteniusaceae & Calatola costaricensis Standl. & Arbóreo & & $\mathrm{LC}$ & Ambas \\
\hline Moraceae & Ficus bullenei I.M. Johnst. & Arbóreo & & & $\mathrm{DM}$ \\
\hline Moraceae & Maquira guianensis Aubl. & Arbóreo & & & Ambas \\
\hline Moraceae & Sorocea sarcocarpa Lanj. \& Wess. Boer & Arbóreo & Occidente Ec & $\mathrm{EN}$ & DM \\
\hline Monimiaceae & Mollinedia ovata Ruiz \& Pav. & Arbustivo & & & LA \\
\hline Myrtaceae & Myrcia splendens (Sw.) DC. & Arbóreo & & & LA \\
\hline Piperaceae & Piper squamulosum C. DC. & Arbóreo & Occidente Ec & & LA \\
\hline Rubiaceae & Pentagonia grandiflora Standl. & Arbóreo & Chocó & & LA \\
\hline Rubiaceae & Psychotria hazenii Standl. & Arbóreo & TCM & & Ambas \\
\hline Rubiaceae & Randia carlosiana $\mathrm{K}$. Krause & Arbóreo & Occidente Ec & $\mathrm{EN}$ & Ambas \\
\hline Rutaceae & Zanthoxylum riedelianum Engl. & Arbóreo & & & LA \\
\hline Sapindaceae & Cupania latifolia Kunth & Arbóreo & & & Ambas \\
\hline Sapotaceae & Chrysophyllum venezuelanense (Pierre) T.D. Penn. & Arbóreo & & & Ambas \\
\hline Urticaceae & Cecropia angustifolia Trécul & Arbóreo & & & LA \\
\hline Urticaceae & Cecropia litoralis Snethlage & Arbóreo & TCM & & DM \\
\hline Urticaceae & Cecropia obtusifolia Bertol. & Arbóreo & & $\mathrm{LC}$ & Ambas \\
\hline Urticaceae & Urera caracasana (Jacq.) Gaudich. ex Griseb. & Arbóreo & & & LA \\
\hline
\end{tabular}


Tabla 2. Índices de diversidad alfa utilizados en el presente estudio, adaptado de: Moreno, 2001; Aguirre, 2013; Campo \& Duval, 2014.

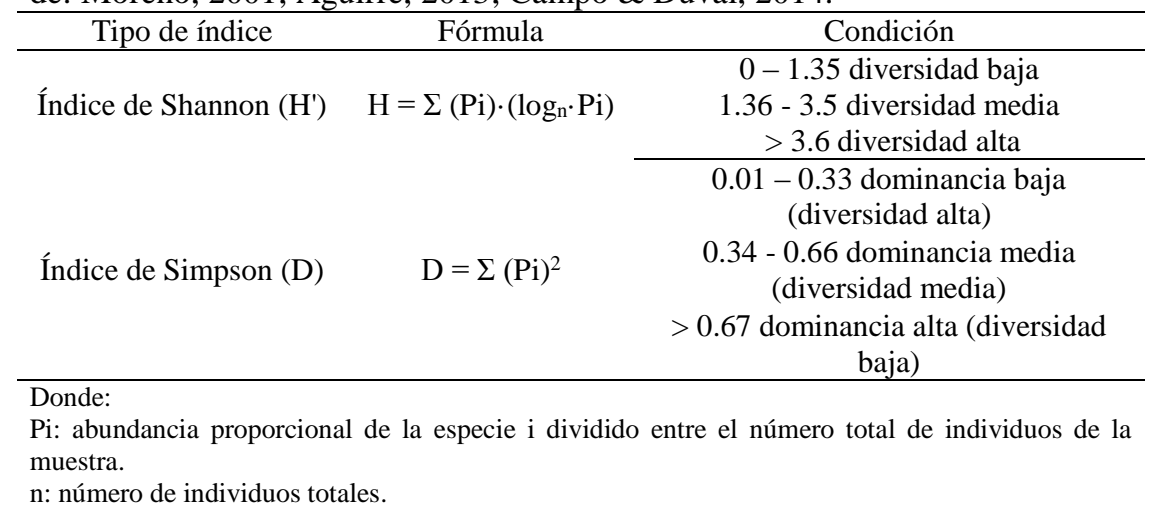

Tabla 3. Índices de diversidad $\alpha$ de la cordillera Chongón Colonche.

\begin{tabular}{llccccccccccc}
\hline Diversidad $\alpha$ & & T1 & T2 & T3 & T4 & T5 & T6 & T7 & T8 & T9 & T10 & Promedio \\
\hline \multirow{5}{*}{ Loma Alta } & Riqueza & 14 & 17 & 12 & 18 & 16 & 16 & 18 & 11 & 15 & 20 & 15.70 \\
& Individuos & 40 & 33 & 28 & 38 & 38 & 41 & 42 & 45 & 34 & 40 & 37.90 \\
& H' & 1.92 & 2.58 & 2.19 & 2.63 & 2.43 & 2.41 & 2.43 & 2.15 & 2.52 & 2.78 & 2.40 \\
& Dominancia & 0.27 & 0.10 & 0.15 & 0.09 & 0.12 & 0.13 & 0.14 & 0.14 & 0.10 & 0.08 & 0.13 \\
& Riqueza & 17 & 16 & 15 & 14 & 18 & 18 & 13 & 12 & 11 & 14 & 14.89 \\
Dos Mangas & Individuos & 56 & 59 & 30 & 32 & 36 & 37 & 37 & 37 & 34 & 32 & 39.78 \\
& H' & 2.22 & 1.98 & 2.48 & 2.44 & 2.70 & 2.62 & 2.19 & 1.97 & 2.02 & 2.36 & 2.30 \\
& Dominancia & 0.17 & 0.24 & 0.10 & 0.10 & 0.08 & 0.09 & 0.15 & 0.21 & 0.18 & 0.13 & 0.15 \\
\hline
\end{tabular}

T1 - T10: Transectos.

H': Diversidad de Shannon-Wiener (estima la heterogeneidad de la comunidad).

nats/ind: Unidad logarítmica de entropía del índice de Shannon - Wiener.

Tabla 4. Análisis de porcentajes de similitud (SIMPER) del bosque de garúa ubicado en dos localidades de la cordillera Chongón Colonche.

\begin{tabular}{|c|c|c|c|c|c|c|}
\hline Localidad & $\begin{array}{c}\text { Promedio } \\
\text { de } \\
\text { similaridad }\end{array}$ & Especies & 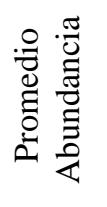 & 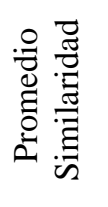 & 毫 & 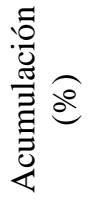 \\
\hline \multirow{6}{*}{ Loma Alta } & \multirow{6}{*}{56.13} & Matisia grandifolia & 3.07 & 11.86 & 21.13 & 21.13 \\
\hline & & Randia carlosiana & 2.12 & 8.18 & 14.57 & 35.70 \\
\hline & & Calatola costaricensis & 1.71 & 6.22 & 11.08 & 46.78 \\
\hline & & Nectandra subbullata & 1.29 & 4.49 & 8.00 & 54.78 \\
\hline & & Chamaedorea linearis & 1.16 & 3.77 & 6.72 & 61.5 \\
\hline & & Dussia lehmannii & 1.18 & 3.64 & 6.48 & 67.98 \\
\hline \multirow{8}{*}{ Dos Mangas } & \multirow{8}{*}{61.80} & Matisia grandifolia & 3.2 & 12.27 & 19.85 & 19.85 \\
\hline & & Chamaedorea linearis & 2.51 & 10.41 & 16.85 & 36.70 \\
\hline & & Psychotria hazenii & 1.46 & 5.18 & 8.37 & 45.08 \\
\hline & & Randia carlosiana & 1.31 & 4.84 & 7.82 & 52.9 \\
\hline & & Maquira guianensis & 1.12 & 4.04 & 6.53 & 59.43 \\
\hline & & Grias peruviana & 1.26 & 4.00 & 6.47 & 65.91 \\
\hline & & Chrysochlamys dependens & 1.07 & 3.94 & 6.38 & 72.29 \\
\hline & & Nectandra subbullata & 1.11 & 3.28 & 5.31 & 77.60 \\
\hline
\end{tabular}

Se muestran los resultados de las especies que contribuyeron por encima de los $5 \%$ a la similitud de cada localidad ordenadas en función de su porcentaje de contribución. 
Tabla 5. Análisis SIMPER, basado en la disimilitud, del bosque de garúa ubicado en dos localidades de la cordillera Chongón Colonche.

\begin{tabular}{lcccr}
\hline Especie & $\begin{array}{c}\text { Disimilitud } \\
\text { Promedio }(\%)\end{array}$ & Dis./DE & Contribución (\%) & Acumulación $(\%)$ \\
\hline Chamaedorea linearis & 3.1 & 1.7 & 5.72 & 5.72 \\
Grias peruviana & 2.93 & 1.68 & 5.4 & 11.12 \\
Calatola costaricensis & 2.24 & 1.32 & 4.13 & 15.25 \\
Matisia grandifolia & 2.11 & 1.39 & 3.89 & 19.14 \\
Dussia lehmannii & 1.97 & 1.35 & 3.62 & 22.77 \\
Chrysochlamys dependens & 1.93 & 1.55 & 3.55 & 26.32 \\
Randia carlosiana & 1.91 & 1.44 & 3.52 & 29.83 \\
Psychotria hazenii & 1.88 & 1.25 & 3.47 & 33.30 \\
Maquira guianensis & 1.84 & 1.35 & 3.39 & 36.69 \\
Carapa guianensis & 1.83 & 1.18 & 3.38 & 40.07 \\
\hline
\end{tabular}

Disimilitud promedio entre grupos: 54.27

Se muestran sólo las diez primeras especies responsables del $40 \%$ acumulado.

Tabla 6. Estructura del bosque de Loma Alta. Se muestra la abundancia, frecuencia, dominancia y el Índice de Valor de Importancia (IVI) al 100\%.

\begin{tabular}{|c|c|c|c|c|c|c|c|}
\hline \multirow{2}{*}{ ESPECIES } & \multicolumn{2}{|c|}{ ABUNDANCIA } & \multicolumn{2}{|c|}{ FRECUENCIA } & \multicolumn{2}{|c|}{ DOMINANCIA } & \multirow{2}{*}{$\begin{array}{l}\text { IVI al } \\
100 \%\end{array}$} \\
\hline & $\mathrm{a}$ & $\mathrm{r}$ & a & $\mathrm{r}$ & $\mathrm{a}$ & $\mathrm{r}$ & \\
\hline Rhodostemonodaphne kunthiana (Nees) Rohwer & 4 & 1.06 & 3 & 1.94 & 8.86 & 50.08 & 17.69 \\
\hline Matisia grandifolia Little & 99 & 26.12 & 10 & 6.45 & 1.60 & 9.06 & 13.88 \\
\hline Randia carlosiana $\mathrm{K}$. Krause & 47 & 12.40 & 10 & 6.45 & 0.40 & 2.29 & 7.05 \\
\hline Handroanthus chrysanthus (Jacq.) S. O. Grose & 2 & 0.53 & 2 & 1.29 & 3.00 & 16.95 & 6.26 \\
\hline Calatola costaricensis Standl. & 32 & 8.44 & 10 & 6.45 & 0.53 & 3.02 & 5.97 \\
\hline Dussia lehmannii Harm & 18 & 4.75 & 8 & 5.16 & 0.93 & 5.27 & 5.06 \\
\hline Nectandra subbullata Rohwer & 19 & 5.01 & 9 & 5.81 & 0.50 & 2.83 & 4.55 \\
\hline Cupania latifolia Kunth & 12 & 3.17 & 6 & 3.87 & 0.58 & 3.28 & 3.44 \\
\hline Chamaedorea linearis (Ruiz \& Pav.) Mart. & 17 & 4.49 & 8 & 5.16 & 0.04 & 0.23 & 3.29 \\
\hline Psychotria hazenii Standl. & 17 & 4.49 & 7 & 4.52 & 0.04 & 0.21 & 3.07 \\
\hline Beilschmiedia alloiophylla (Rusby) Kosterm. & 12 & 3.17 & 6 & 3.87 & 0.35 & 1.97 & 3.00 \\
\hline Gustavia serrata S.A Mori & 9 & 2.37 & 6 & 3.87 & 0.05 & 0.25 & 2.17 \\
\hline Annona oligocarpa R.E. Fr. & 9 & 2.37 & 5 & 3.23 & 0.04 & 0.23 & 1.95 \\
\hline Urera caracasana (Jacq.) Gaudich. ex Griseb. & 8 & 2.11 & 5 & 3.23 & 0.00 & 0.01 & 1.78 \\
\hline Tetrorchidium andinum Müll. Arg. & 7 & 1.85 & 4 & 2.58 & 0.11 & 0.62 & 1.68 \\
\hline Garcinia madruno (Kunth) B. Hammel & 5 & 1.32 & 5 & 3.23 & 0.06 & 0.37 & 1.64 \\
\hline Tabernaemontana amygdalifolia Jacq. & 6 & 1.58 & 5 & 3.23 & 0.01 & 0.08 & 1.63 \\
\hline Maquira guianensis Aubl. & 4 & 1.06 & 4 & 2.58 & 0.16 & 0.93 & 1.52 \\
\hline Cecropia obtusifolia Bertol. & 6 & 1.58 & 4 & 2.58 & 0.01 & 0.06 & 1.41 \\
\hline Acalypha cuneata Poepp. & 5 & 1.32 & 4 & 2.58 & 0.01 & 0.03 & 1.31 \\
\hline Piper squamulosum C. DC. & 4 & 1.06 & 4 & 2.58 & 0.00 & 0.03 & 1.22 \\
\hline Chrysophyllum venezuelanense (Pierre) T.D. Penn. & 3 & 0.79 & 3 & 1.94 & 0.07 & 0.40 & 1.04 \\
\hline Chrysochlamys dependens Planch. \& Triana & 4 & 1.06 & 3 & 1.94 & 0.02 & 0.09 & 1.03 \\
\hline Pentagonia grandiflora Standl. & 4 & 1.06 & 3 & 1.94 & 0.02 & 0.11 & 1.03 \\
\hline Bactris setulosa H. Karsten & 3 & 0.79 & 3 & 1.94 & 0.03 & 0.16 & 0.96 \\
\hline Alchornea leptogyna Diels & 3 & 0.79 & 3 & 1.94 & 0.01 & 0.04 & 0.92 \\
\hline Cecropia angustifolia Trécul & 2 & 0.53 & 2 & 1.29 & 0.14 & 0.80 & 0.87 \\
\hline Aegiphila alba Moldenke & 3 & 0.79 & 2 & 1.29 & 0.06 & 0.32 & 0.80 \\
\hline Cordia collococca $\mathrm{L}$. & 3 & 0.79 & 2 & 1.29 & 0.00 & 0.02 & 0.70 \\
\hline Inga carinata T.D. Penn. & 2 & 0.53 & 2 & 1.29 & 0.00 & 0.01 & 0.61 \\
\hline Vasconcellea microcarpa (Jacq.) A. DC. & 2 & 0.53 & 2 & 1.29 & 0.00 & 0.01 & 0.61 \\
\hline Mollinedia ovata Ruiz \& Pav. & 3 & 0.79 & 1 & 0.65 & 0.00 & 0.02 & 0.49 \\
\hline Zanthoxylum riedelianum Engl. & 2 & 0.53 & 1 & 0.65 & 0.02 & 0.11 & 0.43 \\
\hline Dendropanax macrocarpus Cuatrec. & 1 & 0.26 & 1 & 0.65 & 0.01 & 0.05 & 0.32 \\
\hline Myrcia splendens (Sw.) DC. & 1 & 0.26 & 1 & 0.65 & 0.01 & 0.04 & 0.32 \\
\hline Capparidastrum bonifazianum Cornejo \& Iltis & 1 & & 1 & 0.65 & 0.00 & 0.02 & 0.31 \\
\hline Total & 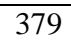 & 100 & 5 & 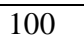 & 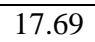 & 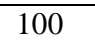 & - \\
\hline
\end{tabular}


Tabla 7. Estructura del bosque de Dos Mangas. Se muestra la abundancia, frecuencia, dominancia y el Índice de Valor de Importancia (IVI) al 100\%. a = absoluta; $r=$ relativa.

\begin{tabular}{|c|c|c|c|c|c|c|c|}
\hline \multirow{2}{*}{ ESPECIES } & \multicolumn{2}{|c|}{ ABUNDANCIA } & \multicolumn{2}{|c|}{ FRECUENCIA } & \multicolumn{2}{|c|}{ DOMINANCIA } & \multirow{2}{*}{$\begin{array}{l}\text { IVI al } \\
100 \%\end{array}$} \\
\hline & $\mathrm{a}$ & $\mathrm{r}$ & $\mathrm{a}$ & $\mathrm{r}$ & $\mathrm{a}$ & $\mathrm{r}$ & \\
\hline Ficus bullenei I.M. Johnst. & 5 & 1.28 & 4 & 2.7 & 17.23 & 45.29 & 16.42 \\
\hline Matisia grandifolia Little & 111 & 28.46 & 10 & 6.76 & 1.7 & 4.47 & 13.23 \\
\hline Maquira guianensis Aubl. & 15 & 3.85 & 9 & 6.08 & 5.97 & 15.69 & 8.54 \\
\hline Nectandra subbullata Rohwer & 18 & 4.62 & 8 & 5.41 & 5.38 & 14.13 & 8.05 \\
\hline Chamaedorea linearis (Ruiz \& Pav.) Mart. & 65 & 16.67 & 10 & 6.76 & 0.23 & 0.61 & 8.01 \\
\hline Cupania latifolia Kunth & 11 & 2.82 & 7 & 4.73 & 2.57 & 6.76 & 4.77 \\
\hline Psychotria hazenii Standl. & 25 & 6.41 & 9 & 6.08 & 0.04 & 0.11 & 4.2 \\
\hline Grias peruviana Miers & 21 & 5.38 & 8 & 5.41 & 0.36 & 0.96 & 3.92 \\
\hline Randia carlosiana $\mathrm{K}$. Krause & 20 & 5.13 & 9 & 6.08 & 0.15 & 0.39 & 3.87 \\
\hline Chrysochlamys dependens Planch. \& Triana & 13 & 3.33 & 9 & 6.08 & 0.05 & 0.14 & 3.18 \\
\hline Beilschmiedia alloiophylla (Rusby) Kosterm & 4 & 1.03 & 3 & 2.03 & 1.72 & 4.51 & 2.52 \\
\hline Calatola costaricensis Standl. & 9 & 2.31 & 7 & 4.73 & 0.14 & 0.37 & 2.47 \\
\hline Carapa guianensis Aubl. & 11 & 2.82 & 6 & 4.05 & 0.12 & 0.33 & 2.4 \\
\hline Dussia lehmannii Harms & 7 & 1.79 & 5 & 3.38 & 0.78 & 2.04 & 2.4 \\
\hline Parinari romeroi Prance & 6 & 1.54 & 5 & 3.38 & 0.65 & 1.71 & 2.21 \\
\hline Acalypha cuneata Poepp. & 9 & 2.31 & 5 & 3.38 & 0.03 & 0.08 & 1.92 \\
\hline Inga carinata T.D. Penn. & 6 & 1.54 & 4 & 2.7 & 0.1 & 0.27 & 1.5 \\
\hline Chrysophyllum venezuelanense (Pierre) T.D. Penn. & 6 & 1.54 & 4 & 2.7 & 0.06 & 0.15 & 1.46 \\
\hline Aegiphila alba Moldenke & 5 & 1.28 & 4 & 2.7 & 0.09 & 0.23 & 1.41 \\
\hline Cordia collococca $\mathrm{L}$. & 5 & 1.28 & 4 & 2.7 & 0.07 & 0.2 & 1.39 \\
\hline Dendropanax macrocarpus Cuatrec. & 4 & 1.03 & 4 & 2.7 & 0.08 & 0.21 & 1.31 \\
\hline Phytelephas aequatorialis Spruce & 3 & 0.77 & 3 & 2.03 & 0.33 & 0.88 & 1.22 \\
\hline Sorocea sarcocarpa Lanj. \& Wess. Boer & 2 & 0.51 & 2 & 1.35 & 0.01 & 0.03 & 0.63 \\
\hline Anemopaegma puberulum (Seibert) Miranda & 2 & 0.51 & 2 & 1.35 & 0 & 0.01 & 0.62 \\
\hline Cecropia litoralis Snethlage & 1 & 0.26 & 1 & 0.68 & 0.06 & 0.15 & 0.36 \\
\hline Rauvolfia tetraphylla $\mathrm{L}$. & 1 & 0.26 & 1 & 0.68 & 0.05 & 0.13 & 0.35 \\
\hline Cecropia obtusifolia Bertol. & 1 & 0.26 & 1 & 0.68 & 0.02 & 0.06 & 0.33 \\
\hline Tetrorchidium andinum Müll. Arg. & 1 & 0.26 & 1 & 0.68 & 0.02 & 0.06 & 0.33 \\
\hline Geonoma undata Klotzsch & 1 & 0.26 & 1 & 0.68 & 0.01 & 0.01 & 0.32 \\
\hline Mauria heterophylla Kunth & 1 & 0.26 & 1 & 0.68 & 0.01 & 0.02 & 0.32 \\
\hline Tanaecium pyramidatum (Rich.) L.G. Lohmann & 1 & 0.26 & 1 & 0.68 & 0 & 0 & 0.31 \\
\hline TOTAL & 390 & 100 & 148 & 100 & 38.05 & 100 & 100 \\
\hline
\end{tabular}

${ }^{1}$ Research Center / Universidad Espíritu Santo. Km 2.5 vía La Puntilla, Samborondón / Ecuador eveavesecuador@gmail.com (*autor corresponsal) https://orcid.org/0000-0003-0006-2730.

2 Jardín Botánico de Guayaquil, Av. Fco. de Orellana y calle 24-N NE, Cdla. Las Orquídeas - Guayaquil / Ecuador. iperez40@hotmail.com.

3 Facultad de Ciencias del Mar / Universidad Estatal Península de Santa Elena. La Libertad / Ecuador. luis.troccoli@gmail.com.

${ }^{4}$ Universidad Científica del Sur \Carrera de Biología Marina. Lima / Perú. haponte @ cientifica.edu.pe.

${ }^{5}$ Escuela de Ingeniería Textil y Confecciones / Facultad de Ingeniería Industrial / Universidad Nacional Mayor de San Marcos. Calle Germán Amézaga N 375, Lima / Perú. otinoco@unmsm.edu.pe. 